\title{
Modified Efficient and Secure Dynamic ID-Based User Authentication Scheme
}

\author{
Toan-Thinh Truong ${ }^{1}$, Minh-Triet $\operatorname{Tran}^{1}$, and Anh-Duc Duong ${ }^{2}$ \\ 1 University of Science, VNU-HCM \\ \{tthinh,tmtriet\}@fit.hcmus.edu.vn \\ 2 University of Information Technology, VNU-HCM \\ daduc@uit.edu.vn
}

\begin{abstract}
Communication is necessary operations in wireless environments. Therefore, we must have a secure remote authentication to defend transactions against illegitimate adversaries in such risky channel. Smart card is one of methods that many schemes used due to its convenience. Recently, Khurram Khan has proposed an enhancement scheme using smart card to recover some pitfalls in Wang et al.'s scheme. They claimed that their scheme remedy those security flaws. Nevertheless, we point out that Khan et al.'s scheme cannot protect user's anonymity. Besides, it does not achieve secret key forward secrecy and cannot resist denial of service attack due to values stored in server's database. Consequently, we present an improvement to their scheme to isolate such problems.
\end{abstract}

Keywords: Mutual authentication, Dynamic identity, Anonymity.

\section{Introduction}

In network environment, users can access services via different devices, for example, PC, laptop, mobile phone. Communication between those devices and services can be operated with many network technologies, such as, wireless, 3G.

There are many methods of constructing secure authentication. In 1981, Lamport [1] is the first person applying cryptographic hash function in authentication. Later, many author also use this approach in their protocols. Typically, there are protocols of Cheng-Chi Lee [2] and Jau-Ji Shen [3]. These authors follow Lamport's approach with slight difference that they use identity to authenticate instead of password table. In 2004, Das et al proposed a scheme [4]. Their scheme has three main advantages. Firstly, it allows users to change password freely. Moreover, it does not maintain a verification table which is used to check login message. Finally, the scheme's security is based on secure one-way hash function. In 2005, I-En Liao [5] discovered Das's scheme not only cannot resist some basic kinds of attacks such as password-guessing but also do not provide mutual authentication. Furthermore, in Das's scheme, password is transmitted in plain-text form at registration phase. Therefore, it is easy to be stolen by server. In I-En Liao's scheme, author use hash function with password before transmitting it to server. So, even server do not know actual user's password. Nonetheless, 
with hash function, I-En Liao's scheme is also vulnerable to password-guessing attack. Consequently, E-J Yoon proposed an improvement [6] to I-En Liao's. In [6], author utilizes random value with password when hashing. So, this causes attacker not to be able to guess true user's password. Recently, Khuram Khan with E-J Yoon's approach devised a protocol [7]. In [7], authors also distribute common secret information to all users and use timestamp to resist replay attack. They also claimed their scheme can protect user's anonymity. In this paper, we prove that their scheme has inability to defend anonymity. Furthermore, it also cannot achieve secret key forward secrecy and does not withstand denial of service attack due to values stored in database's server. Ultimately, we propose an improved version to recover problems mentioned.

The remainder of this paper is organized as follows: section 2 quickly reviews Khan's scheme and discusses its weaknesses. Then, our proposed scheme is presented in section 3, while section 4 discusses the security and efficiency of the proposed scheme. Our conclusions are presented in section 5.

\section{Review and Cryptanalysis of Khurram Khan's Scheme}

In this section, we review Khan's scheme [7] and show his scheme cannot obtain secret key forward secrecy and doesn't protect user's anonymity.

\subsection{Review of Khurram Khan's Scheme}

Their scheme includes five phases. Some important notations are listed as follow:

- $U_{i}, S$ : Qualified user \& remote server.

- $p w_{i}, h($.$) : Unique password of U_{i}$ and one-way hash function.

$-x, y$ : The first \& the second secret keys of the remote server.

- $T, N$ : The timestamp \& the number of times a user registers.

- SC, SK: The smart card \& the session key.

$-\oplus, \|$ : The exclusive-or operation \& concatenation operation.

Registration Phase. $U_{i}$ submits $I D_{i} \& h\left(r \| p w_{i}\right)$ to $S$ via a secure channel, where $r$ is a random value chosen by user. Then, $S$ performs the following steps.

1. $S$ checks $U_{i}$ 's registration credentials \& checks $I D_{i}$ 's existence. If it already exists \& $N$ is equal to $0, S$ requests $U_{i}$ to choose another $I D_{i}$. Otherwise, $S$ computes $J=h\left(x \| I D_{U}\right)$ where $I D_{U}=\left(I D_{i} \| N\right)$ and $L=J \oplus R P W$.

2. $S$ issues $S C$ with $\{L, y\}$ to $U_{i}$ over secure channel. Then, $U_{i}$ stores $r$ in $S C$.

Login Phase. After receiving $S C$ from $S, U_{i}$ uses it to login into $S$.

1. $U_{i}$ inserts $S C$ into another terminal's card-reader. Then he keys $p w_{i} \& I D_{i}$.

2. $S C$ computes $R P W=h\left(r \| p w_{i}\right) \& J=L \oplus R P W$. Then, $S C$ acquires $T_{i}$ \& computes $C_{1}=h\left(T_{i} \| J\right)$.

3. $S C$ generates a random value $d \&$ computes $A I D_{i}=I D_{i} \oplus h\left(y\left\|T_{i}\right\| d\right)$. Finally, $S C$ sends login message $m=\left\{A I D_{i}, T_{i}, d, C_{1}\right\}$ to $S$. 
Authentication Phase. $S$ authenticates the users login request.

1. Verifies the validity of time interval between $T_{i} \& T^{\prime}$. If $\left(T^{\prime}-T_{i}\right) \geq \Delta T$, where $\Delta T$ denotes the expected valid time interval for transmission delay. If this holds, then $S$ rejects \& terminates the session.

2. Computes $I D_{i}=A I D_{i} \oplus h\left(y\left\|T_{i}\right\| d\right)$ \& checks if $I D_{i}$ is valid, otherwise terminates the operation. Then $S$ checks $N$ in database \& computes $I D_{U}=$ $\left(I D_{i} \| N\right), J=h\left(x \| I D_{U}\right)$. Next $S$ checks if $h\left(T_{i} \| J\right) ?=C_{1}$. If this holds, it means $U_{i}$ is an authentic user, whereas the login request is rejected.

3. $S$ acquires $T_{S}$, computes $C_{2}=h\left(C_{1} \oplus J \oplus T_{S}\right) \&$ sends $\left\{C_{2}, T_{S}\right\}$ to $U_{i}$.

4. When receiving message from $S, U_{i}$ checks time interval between $T_{S} \&$ $T^{\prime \prime}$, where $T^{\prime \prime}$ is the timestamp when mutual authentication message was received. If $\left(T^{\prime \prime}-T_{S}\right) \geq \Delta T$, then $U_{i}$ rejects this message \& terminates the session. Otherwise, $U_{i}$ checks if $h\left(C_{1} \oplus J \oplus T_{S}\right) ?=C_{2}$. If this doesn't holds, $U_{i}$ terminates session. Otherwise $U_{i} \& S$ share $S K=h\left(C_{2} \oplus J\right)$.

Password Change Phase. In this phase, $U_{i}$ can change his or her password.

1. $U_{i}$ inserts $S C$ into card-reader \& inputs $I D_{i} \& p w_{i}$.

2. $S C$ computes $R P W^{*}=h\left(r \| p w_{i}\right) \& J^{*}=L \oplus R P W^{*}$. If $J ?=J^{*}$ holds, then $U_{i}$ is allowed to update password. Otherwise, this phase is terminated.

3. $S C$ computes $L=J \oplus R P W \oplus R P W^{*} \oplus h\left(r \| p w^{\prime}{ }_{i}\right) \&$ replaces the old value of $L$ with the new value. Now, the new password is updated.

Lost Smart Card Revocation Phase. $U_{i}$ performs some steps to revoke $S C$.

1. $S$ checks secret credentials's $U_{i}$, e.g. date of birth, identity card number.

2. $S$ changes the value of $N$ to revoke $S C$. In every case of stolen or lost of $S C$, $N$ is increased by one. Later, $U_{i}$ can re-register to $S$ without changing $I D_{i}$.

3. $S$ requests $U_{i}$ to return to registration phase. Here, $U_{i}$ is strongly recommended not to use any previous values for new registration, e.g. password \& random value, otherwise anybody can impersonation $U_{i}$ by using the same credentials previously saved in the lost or stolen $S C$.

\subsection{Cryptanalysis of Muhammad Khurram Khan's Scheme}

- Inability To Protect User's Anonymity: Khan et al claimed that only $S$ can recover $I D_{i}$ of $U_{i}$ due to $y$ used to hide the user's identity during transmission of login message. Hence, adversaries cannot identify the person trying to login into $S$. We see this explanation is not approriate because anyone being a valid user can know $y$. For example, another valid user captures $\left\{A I D_{i}\right.$, $\left.T_{i}, d, C_{1}\right\}$. Then, he computes $I D_{i}=A I D_{i} \oplus h\left(y\left\|T_{i}\right\| d\right)$

- Secret key forward secrecy: Khan et al claimed that even if the server's $x$ \& $y$ happens to be compromised, an adversary cannot impersonate legitimate users by using the revealed keys, because he cannot compute $A I D_{i} \& C_{1}$ in the login message without knowledge of the user's $I D_{i}, p w_{i}, r \& I D_{U}$. In this subsection, we will prove his claim is not true. 
1. With $y$, adversary $A$ can capture any login message $\&$ compute $I D_{i}$ of any user by performing $I D_{i}=A I D_{i} \oplus h\left(y\left\|T_{i}\right\| d\right)$.

2. $A$ creates a new login message to impersonate $U_{i}$. Firstly, $A$ picks $T_{A}$, random value $d_{A}$. Then, $A$ computes $A I D_{i}=I D_{i} \oplus h\left(y\left\|T_{A}\right\| d_{A}\right)$.

3. $A$ assumes $N=0 \&$ computes $J=h\left(x \| I D_{U}\right)$, where $I D_{U}=\left(I D_{i} \| N\right)$

4. $A$ computes $C_{A}=h\left(T_{A} \| J\right)$ and sends $\left\{A I D_{i}, T_{A}, d_{A}, C_{A}\right\}$ to $S$.

5. If everything is alright, $A$ successfully impersonates $U_{i}$. Otherwise, $A$ turns back to step 3 with increasing $N$ by one \& continues later steps.

- Denial of service attack: In Khan's scheme, passwords are not stored at the verification server. However, this scheme stores $N$ value of $U_{i}$. If these values are modified by attackers, many users cannot login into server. For example, in authentication phase of Khan's scheme, $S$ must compute $J=h\left(x \| I D_{U}\right)$, where $I D_{U}=\left(I D_{i} \| N\right)$. Then, $S$ compares $C_{1}$ with $h\left(T_{i} \| J\right)$. So, if $N$ is modified, $C_{1}$ is not equal to $h\left(T_{i} \| J\right) \& S$ will reject login message.

\section{Proposed Scheme}

Our scheme is also divided into the five phases

\subsection{Registration Phase}

$U_{i}$ submits $I D_{i}, h\left(r \| p w_{i}\right)$, where $r$ is a nonce chosen by $U_{i}$. After receiving $\left\{I D_{i}, h\left(r \| p w_{i}\right)\right\}$ from user via a secure channel, $S$ performs following steps.

1. Checking $I D_{i}$ 's existence. If it existed, $S$ intimates $U_{i}$ to choose another $I D$.

2. Generating a random value $e$ \& computing $J=h(x \| e), P=h(J) \& L=J$ $\oplus R P W$. Then $S$ sends $S C$ containing $\{L, e, P\}$ for $U_{i}$ via a secure channel.

3. $U_{i}$ receives $S C$ \& inputs $r$ into it.

\subsection{Login Phase}

$U_{i}$ inserts $S C$ into card-reader, inputs $I D_{i} \& p w_{i}$ to $\operatorname{login} S$. Then, $S C$ performs:

1. Computing $R P W=h\left(r \| p w_{i}\right) \& J^{*}=L \oplus R P W$.

2. Checking whether $h\left(J^{*}\right) ?=P$. If this holds, $S C$ goes to next step. Otherwise, it terminates the session. Then, $S C$ generates a random value $R_{U} \&$ computes $A I D_{i}=I D_{i} \oplus R_{U}, C_{1}=R_{U} \oplus J^{*} \& M_{1}=h\left(I D_{i}\left\|J^{*}\right\| R_{U}\right)$.

3. Finally, $S C$ sends $\left\{A I D_{i}, C_{1}, M_{1}, e\right\}$ to $S$.

\subsection{Mutual Authentication and Session Key Agreement Phase}

$S$ receives $U_{i}$ 's login message $\left\{A I D_{i}, C_{1}, M_{1}, e\right\}$ and performs following steps.

1. $S$ computes $J^{* *}=h(x \| e), R_{U}=C_{1} \oplus J^{* *} \& I D^{*}{ }_{i}=A I D_{i} \oplus R^{*}$. 
2. $S$ checks identity's validity. If everything isn't alright, $S$ terminates the session, otherwise $S$ checks whether $M_{1} ?=h\left(I D^{*}{ }_{i}\left\|J^{* *}\right\| R^{*} U\right)$. If this doesn't holds, $S$ terminates the session, otherwise $S$ generates a random value $R_{S} \&$ computes $C_{2}=R_{S} \oplus J^{* *}, M_{2}=h\left(R_{S}\left\|J^{* *}\right\| I D^{*}{ }_{i}\right)$. Then $S$ sends $\left\{M_{2}\right.$, $\left.C_{2}\right\}$ to user via a common channel.

3. After receiving $\left\{M_{2}, C_{2}\right\}$ from $S$. $U_{i}$ computes $R^{*}{ }_{S}=C_{2} \oplus J^{*} \&$ check if $M_{2} ?=h\left(R^{*}{ }_{S}\left\|J^{*}\right\| I D_{i}\right)$. If this does not hold, $U_{i}$ terminates the session. Otherwise, $U_{i}$ authenticates $S$ successfully. $U_{i}$ sends $M_{3}=h\left(R_{U} \| R^{*}{ }_{S}\right)$ to $S \&$ computes a session key $S K=h\left(R_{U}\left\|R^{*}{ }_{S}\right\| J^{*} \| I D_{i}\right)$.

4. When receiving $\left\{M_{3}\right\}$ from $U_{i}, S$ checks if $M_{3} ?=h\left(R^{*} U \| R_{S}\right)$. If this does not hold, $S$ terminates the session. Otherwise, $S$ authenticates $U_{i}$ successfully. And $S$ also computes $S K=h\left(R^{*} U\left\|R_{S}\right\| J^{* *} \| I D^{*}{ }_{i}\right)$.

\subsection{Password Update Phase}

When $U_{i}$ wants to change password $p w_{i}$. He can perform following steps:

1. Insert $S C$ into card-reader, input $I D_{i}, p w_{i} \&$ choose a new password $p w_{\text {inew }}$.

2. $S C$ computes $R P W=h\left(r \| p w_{i}\right) \& J^{*}=L \oplus R P W$. Then, $S C$ checks if $h\left(J^{*}\right) ?=P$. If this doesn't holds, $S C$ terminates the session. Otherwise, $S C$ computes $L_{\text {new }}=J^{*} \oplus R P W_{\text {new }}$, where $R P W_{\text {new }}=h\left(r \| p w_{\text {inew }}\right)$

3. Finally, $S C$ replaces $L_{i}$ with $L_{\text {inew }}$.

\subsection{Lost Smart Card Revocation Phase}

We also recommend user not to use any previous values for new $S C$, e.g. password and random value. Following are some steps to perform in this phase:

1. User inputs old $I D_{i}$, new password $p w_{i} \&$ new random value $r$. Then $U_{i}$ sends $\left\{\right.$ credentials, $\left.I D_{i}, R P W\right\}$ to $S$ via a secure channel.

2. After receiving this package of message, $S$ checks $I D_{i}$ 's validity in database. If it does not exist, $S$ terminates the session. Otherwise, $S$ continues to check $U_{i}$ 's credentials. If everything is alright, $S$ generates a new random value $e$.

3. $S$ computes $J=h(x \| e), L=J \oplus R P W \& P=h(J)$. Then $S$ sends new $S C$ containing $\{L, e, P\}$ to $U_{i}$. Finally, $U_{i}$ updates random value $r$ into $S C$.

\section{Security and Efficiency Analysis}

In this section, we analyze our scheme on two aspects: security and efficiency.

\subsection{Security Analysis}

1. Replay Attack: In our scheme's authentication phase, if adversary $A$ captures $\left\{A I D_{i}, C_{1}, M_{1}, e\right\}$, he still cannot re-send this package again. For example, $A$ replays the package, but $A$ cannot compute random value $R_{S}$ from $S$ because of lacking value $J$. So, our scheme resists this kind of attack. 
2. User's Anonymity: In our scheme, if adversary $A$ wants to know $I D_{i}$ of user, $A$ must know random value $R_{U}$. However, $R_{U}$ is enrypted with the value $J$ which is not be leaked. Therefore, our scheme can protect user's anonymity.

3. Stolen Verifier Attack: In our scheme, $S$ does not store any user's information except identity, so our scheme can counteract this kind of attack. In our scheme, $S$ generates a random value $e$ for each user. Hence, when authenticating with $S, U_{i}$ only needs to send $e$ to $S$ and $S$ uses master key $x$ to re-construct $h(x \| e)$ of that user. So, $S$ doesn't need to keep $U_{i}$ 's password.

4. Denial of Service Attack: In Khan's scheme, author stores value $N$ of each user. So, if all $N$ in server's database are modified, all users cannot login to server in login phase. Unlike his scheme, our scheme does not store any user's information. Hence, our scheme is immune from this kind of attack.

Besides above attacks, our scheme also has security features similar to Khan's.

\subsection{Efficiency Analysis}

We reuse approach used in some previous schemes to analyze computational complexity. That is, we calculate the number of one-way hash function execution. Let $T_{h}$ be the time to compute one-way hash function. Khan's scheme needs $2 \times T_{h}$ in registration phase, and $8 \times T_{h}$ in login and authentication phases. Our scheme needs $3 \times T_{h}$ in registration phase and $9 \times T_{h}$ in login and authentication phases. Besides, we see his scheme doesn't resist to denial of service attack and cannot protect user's anonymity. Our proposed scheme recovers two pitfalls successfully. However, we don't solve secret key forward secrecy yet.

Table 1. A comparison between our scheme \& Khan's for withstanding various attacks

\begin{tabular}{|c|c|c|}
\hline Schemes & Khan's & Ours \\
\hline Kinds of Attacks & & \\
\hline Denial of service attack & No & Yes \\
\hline User's anonymity & No & Yes \\
\hline Insider attack & Yes & Yes \\
\hline Stolen verification table attack & Yes & Yes \\
\hline Secret key forward secrecy & No & No \\
\hline Mutual authentication \& SK exchange & Yes & Yes \\
\hline Replay attack & Yes & Yes \\
\hline
\end{tabular}

\section{Conclusions}

In this paper, we review Khan's scheme. Although his scheme can withstand some attacks, we see that his scheme is still vulnerable to denial of service attack, secret key forward secrecy and cannot protect user's anonymity. Consequently, we propose an improved scheme to eliminate some problems existing. 


\section{References}

[1] Lamport, L.: Password authentication with insecure communication. Communications of the ACM 24, 770-772 (1981)

[2] Lee, C.-C., Lin, T.-H., Chang, R.-X.: A secure dynamic id based remote user authentication scheme for multi-server environment using smart cards. Expert Syst. Appl. 38(11), 13863-13870 (2011)

[3] Shen, J.-J., Lin, C.-W., Hwang, M.-S.: A modified remote user authentication scheme using smart cards. IEEE Transactions on Consumer Electronics 49, 414-416 (2003)

[4] Das, M.L., Saxena, A., Gulati, V.P.: A dynamic id-based remote user authentication scheme. IEEE Transactions on Consumer Electronics 50(2), 629-631 (2004)

[5] Liao, I.-E., Lee, C.-C., Hwang, M.-S.: Security enhancement for a dynamic idbased remote user authentication scheme. IEEE Transactions on Consumer Electronics 50, 629-631 (2004)

[6] Yoon, E.-J., Yoo, K.-Y.: Improving the Dynamic ID-Based Remote Mutual Authentication Scheme. In: Meersman, R., Tari, Z., Herrero, P. (eds.) OTM 2006 Workshops. LNCS, vol. 4277, pp. 499-507. Springer, Heidelberg (2006)

[7] Khana, M.K., Kimb, S.-K., Alghathbara, K.: Cryptanalysis and security enhancement of a more efficient \& secure dynamic id-based remote user authentication scheme. Computer Communications 34(3), 305-309 (2010) 